(SD) baseline $\mathrm{FEV}_{1}$ was $97.2 \%$ predicted (10.6\%). Treatment difference for the mean change from baseline in $\mathrm{FEV}_{1}$ at Day 29 was 7.0 percentage points $(P=0.0117)$. Treatment difference for the mean change from baseline in sweat chloride at Day 29 was -45.9 $\mathrm{mmol} / \mathrm{L}(P<0.0001)$. Treatment effect for the change from baseline in pooled CFQ-R Respiratory Domain score was 4.1 points versus placebo $(P=0.4020)$. In the ivacaftor period, AEs and SAEs were reported in 13 and 2 subjects, respectively. In the placebo period, AEs and SAEs were reported in 15 and 1 subject, respectively.

Conclusions In CF patients with mild lung disease, ivacaftor improved ventilation inhomogeneity as measured by LCI and respiratory function as measured by percent predicted $\mathrm{FEV}_{1}$. LCI is more sensitive to change in this mildly affected group of subjects and may be a useful outcome measure for future interventional trials in such patients.

\section{S122 IS THERE A GENDER DIFFERENCE IN THE UK CF POPULATION?}

doi:10.1136/thoraxjnl-2012-202678.127

'N Baty, ${ }^{2} S J$ MacNeill, ${ }^{2 P}$ Cullinan, 'EWFW Alton, ${ }^{3} \mathrm{D}$ Bilton, ${ }^{1} \mathrm{U}$ Griesenbach. 'Department of Gene Therapy, Imperial College Londonthe UK CF Gene Therapy Consortium, London, United Kingdom; ' $D$ epartment of Occupational and Environmental Medicine, Imperial College London, London, United Kingdom; ${ }^{3}$ Adult CF Centre, Royal Brompton Hospital, London, United Kingdom

The literature is conflicting with regards to gender differences in the CF population with some studies reporting poorer prognosis in CF females. Variations in study design (cross-sectional $v$ longitudi$\mathrm{nal}$ and single $\mathrm{v}$ multi-centre), study populations (paediatric $\mathrm{V}$ adults) and small sample sizes may in part explain these differences. In an attempt to overcome some of these limitations we analysed the UK CF Trust Registry database, which collates data on the paediatric and adult UK CF population for evidence of gender differences.

Methods We used the 2010 annual review data and compared males and females in age-groups $0-5,6-12,13-15,16-19,20-23$, $24-29$ and $30+$ years for lung function ( $\mathrm{FEV}_{1}$ and $\mathrm{FVC}$ (\% predicted)), infection status, treatments (rhDNase, $\mathrm{O}_{2}$ therapy, Non-Invasive Ventilation (NIV), IV antibiotics, supplementary feeding and pancreatic supplements), body mass index and associated complications (CF related diabetes, osteoporosis, liver disease).

Results Of the 7937 patients attending annual review in 2010 47\% were female. The sex distribution differed by age whereby the proportion of females dropped from 16 years onwards (Figure 1). The mean FEV ${ }_{1}$ was significantly $(p<0.001)$ lower in female teens aged 16-19 years compared to males (females: 71.0 (confidence interval 68.8-73.2), males 76.7 (74.6-78.8), but not in any other age group. Similar results were observed for mean FVC for which females aged $20-23$ yrs also had significantly lower values than males. Teenage female salso had a significantly $(p<0.05)$ higher use of rhDNase, gastrostomies, $\mathrm{O}_{2}$, had more days/yr on IV antibiotics in hospital and had a significantly $(\mathrm{p} \leq 0.001)$ higher rate of CFRD. In addition, a significantly $(p<0.05)$ higher percentage of females older than 13 had more home IV antibiotics. We did not detect any gender differences with respect to chronic bacterial colonisation(PA, SA, Bcepacia and MRSA).

Conclusions There is evidence of a more severe lung function deficit in teenage years. The reduced proportion of females in the adult cohorts is consistent with a survival difference. Further longitudinal analyses will help characterise the identified gender gap and effects on disease progression.

We thank the UK CF Trust for the registry data.

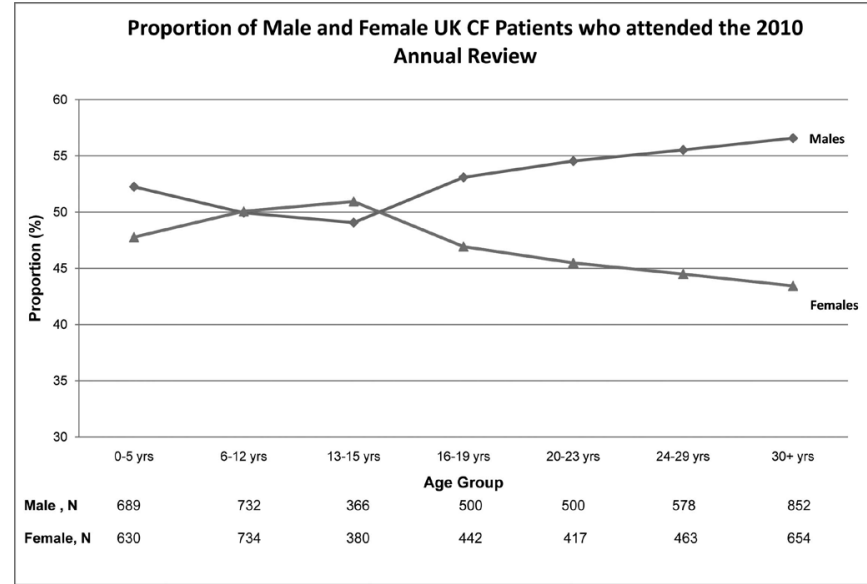

Abstract S122 Figure 1

\section{S123 THE ROLE OF NASAL POTENTIAL DIFFERENCE TESTING IN DIFFICULT CASES OF POSSIBLE CYSTIC FIBROSIS}

doi:10.1136/thoraxjnl-2012-202678.128

'N Simmonds, ${ }^{2} \mathrm{R}$ Pabary, ${ }^{1}$ Y Ya-Tung, 'D Bilton, ${ }^{2}$ EWFW Alton, ${ }^{2} \mathrm{JC}$ Davies. 'Royal Brompton and Harefield NHS Foundation Trust, London, UK; ${ }^{2}$ Imperial College, London, UK

The European CF Diagnostic Working Group guidelines include nasal potential difference (nPD) testing when conventional tests (sweat chloride/CFTR mutation analysis) are inconclusive. This specialist measure of epithelial ion transport is available in relatively few centres throughout the UK and Europe and therefore may not be readily considered as a diagnostic option. We have been offering a clinical service for several years, for both our own patients and those referred in. Here, we report the results of a retrospective audit over a 2.5 year period with an aim to increase awareness and understanding of the test.

Between March 2009 and Sept 2011, 66 patients (58\% female) underwent testing. Median (range) age was 18 (2-67) years. Children under the age of 8 were most commonly tested whilst under anaesthetic for a bronchoscopy. 59\% of patients were referred from other centres. The majority of sweat chloride results (52\%) were in the 30-60 mEq borderline range. 95\% had undergone first-line CFTR mutation analysis: $62 \%$ had none, $37 \%$ had one and 1 subject had 2 mutations, one of which was considered a 'sequence variant'.

One child was unable to tolerate the procedure; $6 \%$ of traces were uninterpretable due to presumed nasal inflammation. Overall, the $\mathrm{nPD}$ results in the context of other features led to a diagnosis of variant/atypical CF in 18\%; abnormality in chloride secretion was more sensitive than sodium absorption. We were able to rule out CF 'to the best of current diagnostic ability' in 60\% including 6 of 8 patients diagnosed with CF in early childhood, but now considered so unlikely to have the disease that re-investigation was warranted. In a further $13 \%$ of cases, nPD was of good quality but was difficult to categorise; in addition to the full CFTR gene sequencing performed routinely, we will investigate these patients for mutations in genes such as $\mathrm{ENaC}$.

Nasal PD was well tolerated and helpful in clarifying the diagnosis in the majority $(78 \%)$ of patients referred. It should be considered in patients for whom, despite careful work up, a diagnosis of $\mathrm{CF}$ is difficult to confirm or rule out.

\section{S124 THE ROLE OF PANCREATIC BETA-CELLS IN THE DIURNAL VARIATION OF GLUCOSE HANDLING IN CYSTIC FIBROSIS}

doi:10.1136/thoraxjnl-2012-202678.129

D Nazareth, K Mohan, M Walshaw. Liverpool Adult CF Unit, Liverpool, United Kingdom

Introduction An impaired glucose tolerance later in the day may be an early sign of diabetes ${ }^{1,2}$, suggesting that there is diurnal variation 